\title{
A very rare case of HPV-53-related cervical cancer, in a 79-year-old woman with a previous history of negative Pap cytology
}

This article was published in the following Dove Press journal:

Clinical Interventions in Aging

15 April 2014

Number of times this article has been viewed

\author{
Roberta Zappacosta' \\ Giuseppe Lattanzio² \\ Patrizia Viola ${ }^{2}$ \\ Manuel Maria Ianieri ${ }^{3}$ \\ Daniela Maria Pia Gatta' \\ Sandra Rosini' \\ 'Cytopathology Unit, Experimental \\ and Clinical Sciences Department, \\ Gabriele d'Annunzio University \\ of Chieti-Pescara, Chieti, Italy; \\ ${ }^{2}$ Surgical Pathology Unit, ${ }^{3}$ Obstetrics \\ and Gynecology Unit, SS Annunziata \\ Hospital, Chieti, Italy
}

\begin{abstract}
The introduction of organized cervical cancer (CC) screening programs has drastically reduced the prevalence of CC. However the incidence is still too high, especially among elderly women. All guidelines strongly recommend a regular Papanicolaou (Pap) testing for young and middle-aged patients. On the other hand, many international professional societies no longer advise screening in women who have undergone hysterectomy, and in women aged 65 years and above, who have a previous history of regular Pap smears. Here we report the case of poorly differentiated $\mathrm{CC}$, involving the pelvic lymph nodes and urinary bladder, occurring in a 79-year-old woman who regularly underwent Pap tests, with no reported cytological abnormalities. In this very rare case, the CC cells, as well as cells from metastatic lymph nodes and cells from urinary specimens, molecularly showed human papilloma virus (HPV)-53. With the limitations of a single case, this report brings important information to prevent CC in elderly patients: the utility of molecular tests to increase sensitivity of Pap smears in postmenopausal women; the importance of HPV-53 as one of the four "emergent" genotypes having a possible role in oncogenesis; and the presence of HPV-53 in lymph node metastases from cervical carcinoma, which would support the role of this virus in the maintenance of malignant status.
\end{abstract}

Keywords: old women, molecular tests, cervical cancer screening, HPV-DNA test, HPV genotyping

\section{Introduction}

Cervical cancer (CC) is the second most common malignancy and the fourth leading cause of cancer mortality among women worldwide. ${ }^{1,2}$ Research has established the incidence peak of $\mathrm{CC}$ in the fourth decade of life, with a median age at diagnosis of 48 years. Approximately $60 \%$ of CC occurs in women over 45 and $20 \%$ in women above 65 years of age. ${ }^{3}$ Certainly, the introduction of organized Papanicolaou (Pap) smear screening programs has resulted in a decreased prevalence of CC by around $70 \%$, but the mortality rate for this neoplasia still remains too high. ${ }^{4,5}$ In particular, the number of elderly patients with $\mathrm{CC}$ is increasing in Europe. ${ }^{6}$

Worldwide, within the older population, the crude incidence of CC is around 17 new cases for every 100,000 females. In the younger population, the corresponding rate ranges from 6 to 7 cases new cases for every $100,000 .^{6}$

Among women over age 65, who were diagnosed with invasive cancer, about $25 \%$ have never been screened by Pap testing, 50\% had no Pap smear in the previous 3 years, and $25 \%$ had Pap screening in the preceding 3 years. $^{7}$

All guidelines strongly recommend regular Pap smears for young and middle-aged women, but no unanimity exists for elderly women. Many international professional societies (such as the American Cancer Societies) no longer advise screening for

\footnotetext{
Correspondence: Roberta Zappacosta Cytopathology Unit, Experimental and Clinical Sciences Department, Gabriele d'Annunzio University of Chieti-Pescara, Via dei Vestini, 66100 Chieti, Italy

Tel +39 87I 357403

Fax +39 87I 540079

Email zappacosta2@hotmail.com
}

submit your manuscript | www.dovepress.com

Dovepress

http://dx.doi.org// 0.2147/CIA.S57294 
patients who have undergone hysterectomy, or for women above 65 years of age with normal exams and proper screening history. ${ }^{8,9}$ In this regard, "proper" screening history is defined as having human papilloma virus (HPV) deoxyribonucleic acid (DNA) test and Pap smear (cotesting) every five years, or cytology alone every three years. ${ }^{9}$

The lack of unanimity about CC screening in the elderly reflects the uncertainty regarding the cost-effectiveness ratio of Pap cytology within the postmenopausal (PMP) population. ${ }^{7}$ The efficacy of cytological screening is known to be lower in higher age groups, when compared with women aged 30-35 years, and is only effective in $20 \%$ of women aging 50 years or older. ${ }^{9}$ A nationwide audit of organized cytological screening in Sweden showed that $25 \%$ of CC involved women with a previous history of normal Pap smears. ${ }^{10}$ In Sweden, over $60 \%$ of patients with cervical squamous carcinoma occurred in PMP women, during 2006. ${ }^{10}$

When the lower efficacy of Pap cytology in the PMP population was first noted, no molecular biomarkers were available to improve screening efficacy. ${ }^{7}$

The involvement of oncogenic HPV (high-risk HPV) in the development of $\mathrm{CC}$ is unequivocal. High-risk HPV infection, with its ability to transform and immortalize infected cells, is a prerequisite of the oncogenesis, although cofactors are needed for malignant transformation. ${ }^{11}$ Consciousness of this led to the development of molecular tests with higher sensitivity compared to cytology. The introduction of a HPV DNA test within CC screening of PMP women, could reduce the incidence of this neoplasia by about $25 \%$ or more. ${ }^{10,12,13}$

Here we report the case of a 79-year-old woman with HPV-53-related CC and a previous history of regular Pap smear screening showing no cytological abnormalities.

\section{The case}

On July 2013, a 79-year-old Caucasian PMP woman presented to the Emergency Department with vaginal bleeding and malodorous vaginal discharge.

Laboratory analysis showed: white blood cell count of $7.6 \times 10^{9} / \mathrm{L}$, with a slight high percentage of neutrophils (69.9\%); red blood cell count of $4.43 \times 10^{12} / \mathrm{L}$; platelet count of $232 \times 10^{9} / \mathrm{L}$; hemoglobin value of $125 \mathrm{~g} / \mathrm{L}$; hematocrit of $39.1 \%$; and a serum albumin value of $57 \mathrm{~g} / \mathrm{L}$. Ferritin and transferrin were both within normal limits. Hepatitis B surface antigen (HBsAg), hepatitis C antibody (HCVAb), and human immunodeficiency virus antibody (HIVAb) were all negative. All tumor markers were within normal ranges.
The patient experienced menarche at age 15, was pregnant three times, and had three children. She reported regular menstrual cycle until 54 years, and no previous history of PMP bleeding. The patient also had a series of normal Pap smears, the last performed the year before. Her medical history showed hypertension, for which she was taking $\beta$-adrenergic blocking agents, and breast cancer, for which she took tamoxifen. No nicotine or alcohol consumption was reported. The patient's father had died of gastric cancer; her sister had undergone total hysterectomy because of ovarian cancer.

On gynecological examination, a large mucosal lesion involving the cervix and the lower third of the vagina was identified. The left parametrial tissue, up to the pelvic side wall, was firm and indurated.

Chest X-ray documented no pathological findings. Magnetic resonance (MR) imaging, before and after intravenous injection of gadopentetate dimeglumine (T1- and T2-weighted sequences), revealed a large and hyperintense mass of $4.5 \mathrm{~cm}$ in maximum diameter, located in the left side of the cervix. After intravenous injection of gadopentetate dimeglumine, the cervical mass, left parametrium, left vaginal fornix, as well as the bladder mucosa demonstrated rapid enhancement. Axial T2-weighted MR images showed hyperintense obturator and internal iliac lymph nodes. Based on MR evidence, the disease was staged as MR International Federation of Gynecology and Obstetrics (FIGO) stage IV CC, ${ }^{14}$ with bladder involvement. Cystoscopy confirmed infiltration of the bladder mucosa.

The patient underwent a radical Wertheim hysterectomy, with bilateral pelvic lymph node dissection.

Histopathological analysis of the uterine cervix showed a poorly differentiated, keratinizing squamous cell carcinoma (Figure 1A), with parametrial and pelvic lymph node involvement (Figure 1B). Microscopic examination of voided urinary samples showed atypical keratinizing squamous cells (Figure 2).

\section{Materials and methods}

This study was approved by the Ethical Committee of G d'Annunzio University, in accordance with the principles outlined in the Declaration of Helsinki. Written informed consent was obtained from the patient.

From the electronic files of the Surgical Pathology Department of the SS Annunziata Hospital, a series of cervical liquid-based cytology (LBC) samples, annually collected from the patient from April 2004 to July 2013, were retrospectively retrieved and reviewed. These specimens 


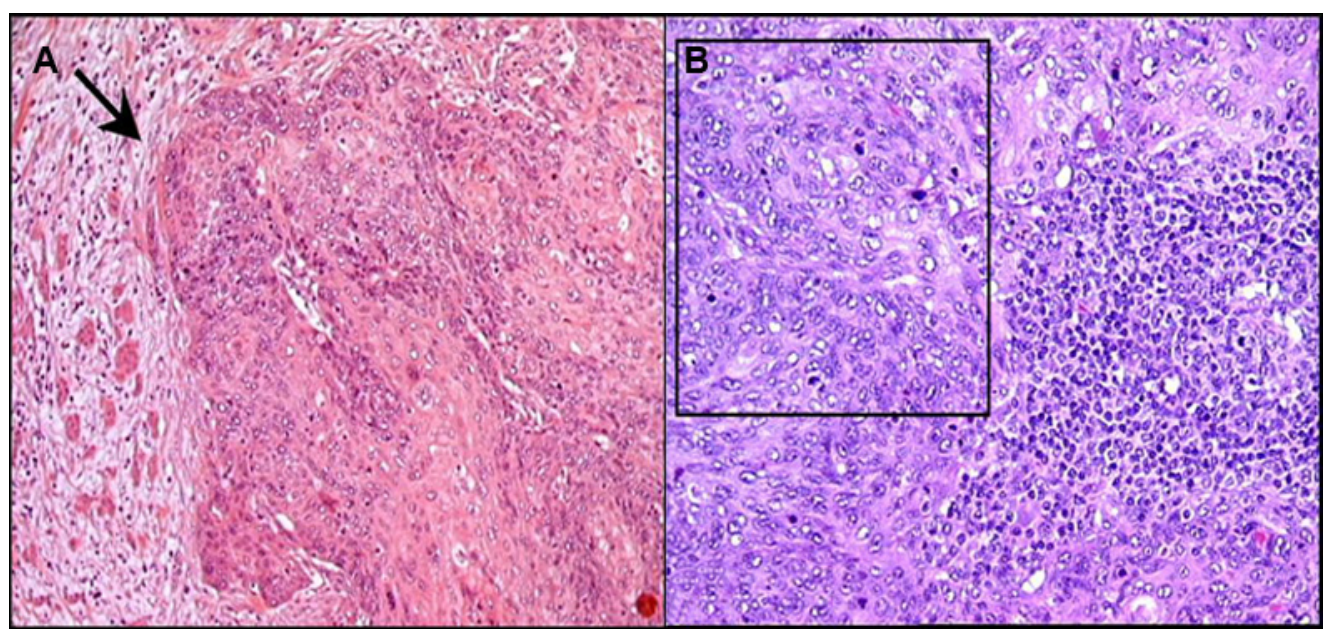

Figure I (A) Histopathological analysis of cervical tissue. Nests of neoplastic squamous cells are invading the stroma (arrow head). The cancer is poorly differentiated and keratinizing (original magnification $\times 200$ ). (B) Pelvic lymph node. Metastatic cells from squamous cell carcinoma (black square) are surrounded by normal lymphocytes (original magnification $\times 200$ ).

had been stored at room temperature and in accordance with the protocol of the Regional Cervical Cytology Biobank, which is located in the Laboratory of Advanced Diagnostic Techniques in Cellular Pathology of Abruzzo Region.

A total of $4 \mathrm{~mL}$ aliquots from each of the cervical cytological samples, and voided urinary samples were removed to perform a Hybrid Capture 2 test (HC2 HPV DNA Test; Qiagen, Venlo, the Netherlands), in accordance to manufacturer's protocol. The HC2 test simultaneously detects 13 oncogenic HPV types (16, 18, 31, 33, 35, 39, $45,51,52,56,58,59$, and 68$)$. The $\mathrm{HC} 2$ reactions, as a chemiluminescent signal, were read by an offline luminometer system (Digene DML 2000 Microplate Luminometer; Qiagen). The luminometer provided a viral load value for each individual sample, comparing the value obtained from each specimen to the mean of a series of positive controls containing $1 \mathrm{pg} / \mathrm{mL}$ of HPV DNA. The amount of $1 \mathrm{pg} / \mathrm{mL}$ of HPV DNA corresponded to $\sim 100,000 \mathrm{HPV}-16$ genomes/mL or 5,000 HPV copies per reaction. The cutoff of 1 RLU was used to classify a specimen as positive or negative.

All the cervical cytological specimens resulted as highrisk HPV-positive.

Formalin-fixed-paraffin-embedded (FFPE) specimens were also evaluated for the presence of HPV. For our purpose, we selected one FFPE tissue sample from CC, one from contiguous cervical intraepithelial neoplasia grade 2- (CIN2-) or worse tissue, one from nonneoplastic cervix, two from metastatic lymph nodes, and two from nonmetastatic lymph nodes. Three sections of $10 \mu \mathrm{m}$ were then cut from each tissue block and processed for DNA extrac- tion. Before sectioning, two outer sections were discarded. In order to prevent possible sample-to-sample contamination, both the microtome blade and working surface were cleaned of tissue and/or paraffin parts and decontaminated using DNA Away ${ }^{\mathrm{TM}}$ solution (DNA Away Surface Decontaminant; Thermo Fisher Scientific Inc, Waltham, MA, USA) after each use.

Tissue sections were incubated with $20 \mu \mathrm{L}$ of proteinase $\mathrm{K}$ overnight, on a rocking platform at $56^{\circ} \mathrm{C}$ and $400 \mathrm{rpm}$.

A $5 \mathrm{~mL}$ volume from each deparaffinized specimen and a $5 \mathrm{~mL}$ for each LBC sample (cervical and urinary ones) were

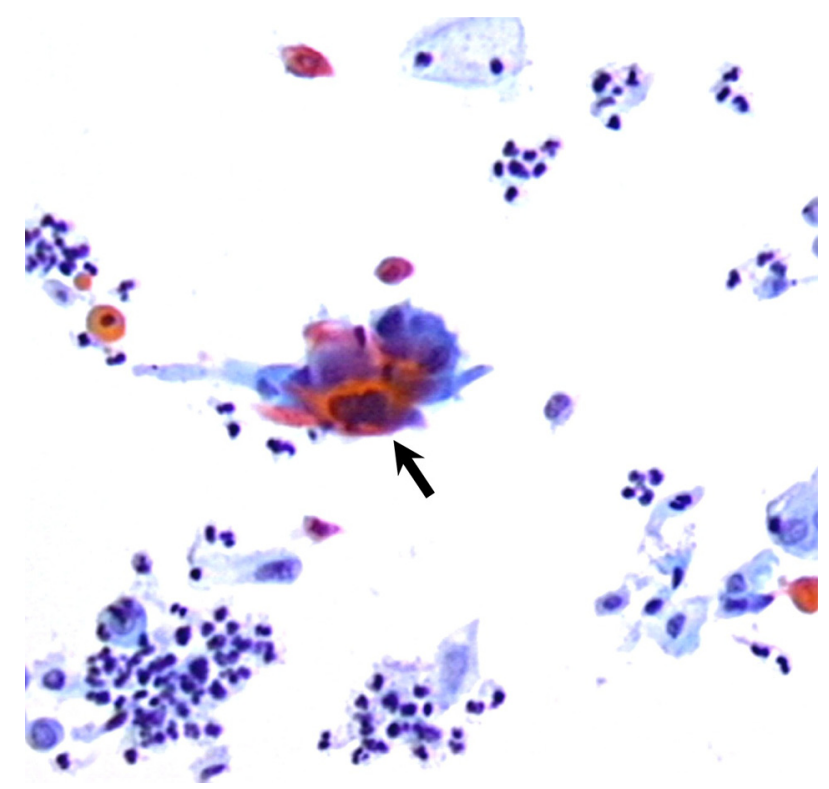

Figure 2 Bladder cytological sample, showing the presence of malignant squamous cells (black arrow) as extrinsic infiltration from cervical carcinoma (original magnification $\times 200$ ). 
then transferred into a fresh $10 \mathrm{~mL}$ tube. Nucleic acids were extracted using silica extraction technology (NucliSENS ${ }^{\circledR}$ easyMAG ${ }^{\circledR}$ automated technology bioMérieux, Craponne, France). Finally, nucleic acids were eluted from the solid phase in $55 \mu \mathrm{L}$ of elution buffer.

HPV genotyping was performed using a semiquantitative highly multiplexed real-time polymerase chain reaction (PCR) kit (Anyplex ${ }^{\mathrm{TM}}$ II HPV28 Detection, Seegene Inc., Seoul, Korea), according to the manufacturer's protocol. The Anyplex II HPV28 detects 19 oncogenic HPVs (16, 18, 26, $31,33,35,39,45,51,52,53,56,58,59,66,68,69,73$, and 82 ) and nine nononcogenic ones $(6,11,40,42,43,44,54,61$, and 70). This technology is based on melting curve analysis PCR on a CFX96 ${ }^{\text {TM }}$ Real-Time PCR Detection System (Bio-Rad, Laboratories Pty, Ltd, Hercules, California, USA). Data interpretation was obtained using Seegene Viewer version 2.0 for CFX96 software (Seegene Inc.).

Anyplex II HPV28 has been validated for use on nucleic acids previously extracted from frozen and FFPE tissue, as well as from LBC specimens, from all anatomical sites. This technology is highly effective on nucleic acids extracted by easyMAG ${ }^{\circledR}$ technology. ${ }^{15}$

\section{Results}

The CC cells showed HPV-53-positive result (Figure 3). The CIN2+ tissue near to the invasive cancer showed HPV-16, $-53,-35,-39,-40,-53,-54,-59,-61,-68$, and -82 . No HPV was detected in the nonneoplastic cervical tissue. The metastatic lymph nodes showed HPV-53; no HPV was found in the nonmetastatic ones. Bladder atypical keratinizing cells also demonstrated a HPV-53-positive result.

\section{Discussion}

In a rapidly growing elderly population, little information is available regarding the occurrence of lower genital tract disease since most studies on $\mathrm{CC}$ have focused on premenopausal women. However, it is certain that PMP women remain susceptible to oncogenic HPV infection. ${ }^{16}$ In this context, the underutilization of screening methods and the low sensitivity of Pap smear test, seems to be the main reasons for the high prevalence of CC in women aged 50 years or older.

Recently a wide range of international studies demonstrated the high sensitivity of HPV DNA testing in the early detection of $\mathrm{CC}$ and its precursors. ${ }^{17}$

In 1999, the US Food and Drug Administration (FDA) approved the use of HPV DNA testing (HC2 technology) within $\mathrm{CC}$ screening programs. It was validated in primary screening of women aged 30 years and older, and as a reflex test to triage equivocal cytology for women of all ages. ${ }^{18-21}$

CC screening in Italy comprises women 25-64 years of age. The screening interval is 3 years. Until 2009, cytology was offered as the primary screening test, with HPV DNA tests being used to triage women with cytological abnormalities. ${ }^{22,23}$ Starting from 2010 , Italy changed its strategy, and the HC2 test was introduced as a primary screening test, in women aging 30-64 years. ${ }^{24}$

As showed by Ronco et al this new screening strategy will prospectively reduce $\mathrm{CC}$ morbidity and mortality. ${ }^{22}$ In addition, women aged 65 and older with negative HPV DNA test results for at least 10 years and with no history of CIN2 will not be screened any longer.

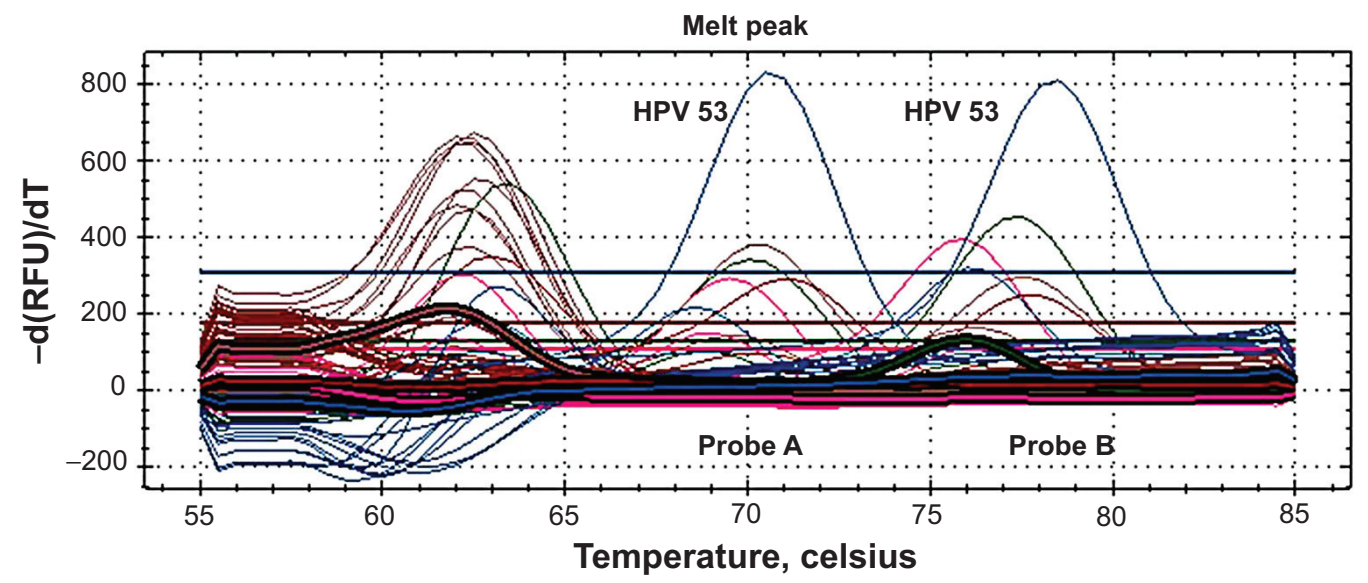

Figure 3 Real-time PCR performed on cervical cancer cells.

Notes: Anyplex ${ }^{\text {TM }}$ II HPV28 detection (Seegene Inc., Seoul, Korea) assay simultaneously detects 28 HPV genotypes. The picture shows the appearance of the higher melting peaks. The Probe A peak demonstrates amplification of plasmid DNA, which is considered to be the positive internal control. The Probe B peak demonstrates the amplification of HPV-53 detected within the cervical cancer cells. $-\mathrm{d}(\mathrm{RFU}) / \mathrm{dT}$ : post-amplification melt-curve analysis, performed by plotting the negative value of the change in rate of fluorescence units against temperature.

Abbreviations: DNA, deoxyribonucleic acid; HPV, human papilloma virus; PCR, polymerase chain reaction. 
Although the present report suffers from the inherent problem of a single case, we believe that our results can bring important information and practical approaches for CC prevention in elderly patients:

1. When HPV acquisition is followed by viral persistence, there is a high probability for progression of precancerous lesions to invasive cancer. In our patient, we retrospectively confirmed the high efficacy of the molecular test in detecting oncogenic infection, despite Pap-negative results. The use of this test could have successfully prevented invasive $\mathrm{CC}$ in this elderly woman.

2. In the lower genital tract, simultaneous infection with multiple HPV genotypes is often observed. Recent literature has shown that HPV type distribution and oncogenicity can be strongly associated with age-related changes within the cervical epithelium. ${ }^{17}$

HPV-16 and - 18 represent about $70 \%$ of all oncogenic HPV types worldwide. Low-prevalence HPV types, such as HPV-52, $-53,-81$, and -83 , are more likely to occur in conjunction with high-prevalence HPV types, such as HPV-16 and $-18 .^{25}$

HPV-53, actually defined as a "probable high-risk type", ${ }^{26}$ is now recognized as one of the four "emergent" genotypes, with a possible role in oncogenesis. HPV-53 infection has been reported in $1.2 \%-16.2 \%$ of women with high-grade cytology but never in patients with $\mathrm{CC} .{ }^{27}$ It has been hypothesized that the true prevalence of HPV-53 is probably underestimated since this genotype has not been included in tests that are frequently in use. A recent report suggests that this genotype be added to $\mathrm{HC} 2$ probes. ${ }^{28}$

In the present case, we molecularly analyzed cervical tissue showing invasive $\mathrm{CC}$, as well as tissue from adjacent areas showing CIN2+ lesion. The CIN2+ tissue revealed ten different HPV types, while the invasive cancer, lymph node metastasis, and atypical urinary cells demonstrated only one genotype: HPV-53.

In our patient, HPV-16 may probably have had a cooperative interaction with HPV-53 in starting neoplastic transformation. It would be likely that HPV-53 maintained the malignant phenotype induced by HPV-16 and subsequently, induced the switch of high-grade intraepithelial lesions into invasive cancer. ${ }^{29}$

3. The detection of HPV DNA in the metastatic lymph nodes of patients with CCs was first reported in 1986, by Lancaster et al. ${ }^{30}$ More recently, other authors have demonstrated that distant metastases from HPV-related tumors can also contain the virus. ${ }^{31}$ On the other hand, a variable proportion of nonmetastatic lymph nodes harboring oncogenic HPV have also been described. Several hypotheses could explain these discrepancies. Firstly, the sensitivity of the technologies used to detect metastatic cells (in situ hybridization versus immunohistochemistry). Secondly, the presence of HPV-DNA in lymph nodal tissue specimens could be the result of damaged non-neoplastic cervical cells, conveyed by lymphatic flow or by phagocyte cells during viral infection, and progressing to lymph nodes even after the clearance of the lesion. ${ }^{32}$

In the present case, using highly sensitive molecular techniques, such as real-time PCR, we did not find HPV within the CC-negative lymph nodes; vice versa, we demonstrated the virus in metastatic nodes, which also showed the same HPV type detected within primary CC (HPV-53). In our opinion, the detection of HPV-53 in metastatic cells far from primary tumor would support the role of this virus in the maintenance of malignant status.

These findings also dispel the suspicion that $\mathrm{CC}$ might have been induced by tamoxifen. Literature shows that the carcinogenic effect of tamoxifen seems to be limited to endocervical glandular epithelium. In the cervical smear of women treated with tamoxifen, a higher incidence of benign reactive atypia or atypical squamous cells of undetermined significance has only been found, without an increased risk of dysplasia or CC. ${ }^{33,34}$

In conclusion, with the limitation of a single case, our report would put the attention of clinicians on the limitation of cervical cytology in PMP women. We strongly believe that the inclusion of the HPV DNA molecular test, possibly with genotyping, in CC prevention strategies would both increase the sensitivity of cancer detection and reduce overtreatment of clinically irrelevant lesions. ${ }^{9,33}$

We certainly know that other factors, such as economic costs, will also affect the decision to screen PMP women. However, it would also be important to focus attention to elaborate age-specific guidelines for $\mathrm{CC}$ prevention, emphasizing the importance of testing the older population with molecular techniques, before discontinuing.

\section{Disclosure}

The authors report no conflicts of interest in this work.

\section{References}

1. Waggoner SE. Cervical cancer. Lancet. 2003;361(9376):2217-2225.

2. Arbyn M, Castellsagué X, de Sanjosé S, et al. Worldwide burden of cervical cancer in 2008. Ann Oncol. 2011;22(12):2675-2686.

3. seer.cancer.gov [homepage on the Internet]. SEER cancer statistics review, 1975-2007. National Cancer Institute; 2010 [updated January 7, 2011] Available from: http://seer.cancer.gov/archive/csr/1975_2007/. Accessed March 24, 2014.

4. Siegel R, Naishadham D, Jemal A. Cancer statistics, 2012. CA Cancer J Clin. 2012;62(1):10-29. 
5. American Cancer Society. Cancer Facts and Figures 2012. Atlanta, GA: American Cancer Society; 2012. Available from: http://www.cancer. org/acs/groups/content/@epidemiologysurveilance/documents/document/acspc-031941.pdf. Accessed March 24, 2014.

6. Meijer CJ, Berkhof J, Castle PE, et al. Guidelines for human papillomavirus DNA test requirements for primary cervical cancer screening in women 30 years and older. Int J Cancer. 2009;124(3):516-520.

7. Sirovich BE, Gottlieb DJ, Fisher ES. The burden of prevention: downstream consequences of Pap smear testing in the elderly. JMed Screen. 2003;10(4):189-195.

8. Committee on Practice Bulletins-Gynecology. ACOG Practice Bulletin Number 131: Screening for cervical cancer. Obstet Gynecol. 2012; 120(5):1222-1238.

9. Saslow D, Solomon D, Lawson HW, et al; American Cancer Society; American Society for Colposcopy and Cervical Pathology; American Society for Clinical Pathology. American Cancer Society, American Society for Colposcopy and Cervical Pathology, and American Society for Clinical Pathology screening guidelines for the prevention and early detection of cervical cancer. Am J Clin Pathol. 2012; 137(4):516-542.

10. Gyllensten U, Gustavsson I, Lindell M, Wilander E. Primary high-risk HPV screening for cervical cancer in post-menopausal women. Gynecol Oncol. 2012;125(2):343-345.

11. Zur Hausen H. Papillomavirus infections - a major cause of human cancers. Biochim Biophys Acta. 2006;1288(2):F55-F78.

12. Gyllensten U, Lindell M, Gustafsson I, Wilander E. HPV test shows low sensitivity of Pap screen in older women. Lancet Oncol. 2010;11(6):509-510; author reply 510.

13. Ko EM, Tambouret R, Wilbur D, Goodman A. HPV reflex testing in menopausal women. Patholog Res Int. 2011;1:4-7.

14. Pecorelli S. Revised FIGO staging for carcinoma of the vulva, cervix, and endometrium. Int J Gynaecol Obstet. 2009;105(2):103-104.

15. Danioth D, Alffolter M, Imeri F, Altwegg M. Multiplex real-time PCR for the simultaneous detection of 7 sexually transmitted pathogens reveals a high rate of multiple infections. Sex Transm Infect. 2013;89(Suppl 1):A106.

16. Castle PE, Schiffman M, Herrero R, et al. A prospective study of age trends in cervical human papillomavirus acquisition and persistence in Guanacaste, Costa Rica. J Infect Dis. 2005;191(11): 1808-1816.

17. Castle PE, Jeronimo J, Schiffman M, et al. Age-related changes of the cervix influence human papillomavirus type distribution. Cancer Res. 2006;66(2):1218-1224.

18. Wright TC Jr, Schiffman M. Adding a test for human papillomavirus DNA to cervical-cancer screening. $N$ Engl J Med. 2003; 348(6):489-490.

19. Saslow D, Runowicz CD, Solomon D, et al; American Cancer Society. American Cancer Society guideline for the early detection of cervical neoplasia and cancer. CA Cancer J Clin. 2002;52(6):342-362.

20. Wright TC, Cox JT, Massad LS, Twiggs LB, Wilkinson EJ; ASCCPSponsored Consensus Conference. 2001 consensus guidelines for the management of women with cervical cytological abnormalities. JAMA. 2002;287(16):2120-2129.

21. ACOG practice bulletin: Clinical management guidelines for obstetrician-gynecologists. Number 45, August 2003. Cervical cytology screening: (replaces committee opinion number 152, March 1995). Obstet Gynecol. 2003;102(2):417-427.

\section{Clinical Interventions in Aging}

\section{Publish your work in this journal}

Clinical Interventions in Aging is an international, peer-reviewed journal focusing on evidence-based reports on the value or lack thereof of treatments intended to prevent or delay the onset of maladaptive correlates of aging in human beings. This journal is indexed on PubMed Central, MedLine,
22. Ronco G, Giorgi-Rossi P, Carozzi F, et al; New Technologies for Cervical Cancer Screening Working Group. Results at recruitment from a randomized controlled trial comparing human papillomavirus testing alone with conventional cytology as the primary cervical cancer screening test. J Natl Cancer Inst. 2008;100(7):492-501.

23. Ronco G, Giorgi-Rossi P, Carozzi F, et al; New Technologies for Cervical Cancer Screening Working Group. Human papillomavirus testing and liquid-based cytology in primary screening of women younger than 35 years: results at recruitment for a randomised controlled trial. Lancet Oncol. 2006;7(7):547-555.

24. Leinonen M, Nieminen P, Kotaniemi-Talonen L, et al. Age-specific evaluation of primary human papillomavirus screening vs conventional cytology in a randomized setting. J Natl Cancer Inst. 2009; 101(23):1612-1623.

25. de Sanjose S, Quint WG, Alemany L, et al; Retrospective International Survey and HPV Time Trends Study Group. Human papillomavirus genotype attribution in invasive cervical cancer: a retrospective crosssectional worldwide study. Lancet Oncol. 2010;11(11):1048-1056.

26. Kocjan BJ, Seme K, Mocilnik T, Jancar N, Vrtacnik-Bokal E, Poljak M. Genomic diversity of human papillomavirus genotype 53 in an ethnogeographically closed cohort of white European women. J Med Virol. 2007;79(4):431-438.

27. Brown DR, Shew ML, Qadadri B, et al. A longitudinal study of genital human papillomavirus infection in a cohort of closely followed adolescent women. J Infect Dis. 2005;191(2):182-192.

28. Dickson EL, Vogel RI, Bliss RL, Downs LS. Multiple-type human papillomavirus (HPV) infections: a cross-sectional analysis of the prevalence of specific types in 309,000 women referred for HPV testing at the time of cervical cytology. Int J Gynecol Cancer. 2013;23(7):1295-1302.

29. Lindemann MLM, Sánchez Calvo JM, Chacón de Antonio J, et al. Prevalence and distribution of high-risk genotypes of HPV in women with severe cervical lesions in Madrid, Spain: importance of detecting genotype 16 and other high-risk genotypes. Adv Prev Med. 2011;2011:1-4.

30. Lancaster WD, Castellano C, Santos C, Delgado G, Kurman RJ, Jenson AB. Human papillomavirus deoxyribonucleic acid in cervical carcinoma from primary and metastatic sites. Am JObstet Gynecol. 1986; 154(1):115-119.

31. Ikenberg H, Teufel G, Schmitt B, Kommoss F, Stanimirovic B, Pfleiderer A. Human papillomavirus DNA in distant metastases of cervical cancer. Gynecol Oncol. 1993;48(1):56-60.

32. Czeglédy J, Iosif C, Forslund O, Willén R, Hansson BG. Detection of human papilloma virus DNA in lymph nodes extirpated at radical surgery for cervical cancer is not predictive of recurrence. J Med Virol. 1998;54(3):183-185.

33. Cox JT, Castle PE, Behrens CM, Sharma A, Wright TC, Cuzick J; Athena HPV Study Group. Comparison of cervical cancer screening strategies incorporating different combinations of cytology, HPV testing, and genotyping for HPV 16/18: results from the ATHENA HPV study. Am J Obstet Gynecol. 2013;208(3):184.e1-184.e11.

34. Gill BL, Simpson JF, Somlo G, McGonigle KF, Wilczynski SP. Effects of tamoxifen on the cytology of the uterine cervix in breast cancer patients. Diagn Cytopathol. 1998;19(6):417-422.

CAS, Scopus and the Elsevier Bibliographic databases. The manuscript management system is completely online and includes a very quick and fair peer-review system, which is all easy to use. Visit http://www.dovepress. com/testimonials.php to read real quotes from published authors. 